\title{
Beam spin asymmetry of neutral pion in semi-inclusive electroproduction
}

\author{
Zhun LU*i \\ Department of Physics, Southeast University, Nanjing, China 211189 \\ E-mail: zhunluaseu.edu.cn \\ Wenjuan Mao \\ Department of Physics, Southeast University, Nanjing, China 211189 \\ E-mail: 230129270 eseu.edu.cn
}

\begin{abstract}
We study the beam spin asymmetry $A_{L U}^{\sin \phi_{h}}$ in semi-inclusive electroproduction of neutral pion contributed by the $T$-odd twist-3 distribution function $g^{\perp}\left(x, \boldsymbol{k}_{T}^{2}\right)$. The distributions for the $u$ and $d$ quarks inside the proton are calculated in a spectator model including the scalar and the axialvector diquark components. Using the model results, we estimate the asymmetry $A_{L U}^{\sin \phi_{h}}$ in $e p \rightarrow$ $e^{\prime} \pi^{0} X$ process in which the lepton beam is longitudinally polarized. The model prediction is compared with the data measured by the CLAS and HERMES Collaborations, showing that our numerical results agree with the experimental data reasonably. We also make a prediction on the beam spin asymmetry in $\pi^{0}$ electroproduction at CLAS12 using the same model calculation.
\end{abstract}

XXI International Workshop on Deep-Inelastic Scattering and Related Subject-DIS2013,

22-26 April 2013

Marseilles, France

\footnotetext{
* Speaker.

${ }^{\dagger}$ This work is supported by NSFC (China) with Grant No. 11005018, by SRF for ROCS from SEM, and by the Fundamental Research Funds for the Central Universities.
} 


\section{Introduction}

Recently substantial single spin asymmetries (SSAs) have been measured in semi-inclusive deep inelastic scattering(SIDIS). In the particular case of a longitudinally polarized beam colliding on an unpolarized target $[1,2,3]$, an asymmetry with $\sin \phi_{h}$ modulation, the so-called beam SSA, emerges. A new source contributing to the beam SSA has been identified, either from the model calculations [4] or from the updated decomposition of the unpolarized quark-quark correlator [5], where the twist-3 TMD $g^{\perp}\left(x, \boldsymbol{k}_{T}^{2}\right)$ plays a crucial role. We present an analysis on the beam SSA $A_{L U}^{\sin \phi_{h}}$ in neutral pion production, based on the effect from $g^{\perp}$. To this end we calculate $g^{\perp}$ for the $u$ and $d$ quarks inside the proton, using a spectator model including both scalar and axial-vector diquarks. We adopt a specific model given in Ref. [6]. Using the calculated $g^{\perp u}$ and $g^{\perp d}$, we estimate the beam SSA in neutral pion production at the kinematics of CLAS, which has measured the beam SSA recently with high precision [3]. We also make a comparison between our calculation and the $\pi^{0}$ data measured by the HERMES Collaboration [2] for further testing. Finally, we present the prediction of $A_{L U}^{\sin \phi_{h}}$ for $\pi^{0}$ production at CLAS12.

\section{2. $g^{\perp}$ of the proton in spectator model with an axial-vector diquark}

The starting point to calculate $g^{\perp}\left(x, \boldsymbol{k}_{T}^{2}\right)$ is the gauge-invariant quark-quark correlator for the unpolarized nucleon

$$
\Phi^{[+]}\left(x, k_{T}\right)=\int \frac{d \xi^{-} d^{2} \xi_{T}}{(2 \pi)^{3}} e^{i k \cdot \xi}\left\langle P\left|\bar{\psi}_{j}(0) \mathscr{L}\left[0^{-}, \infty^{-}\right] \times \mathscr{L}\left[0_{T}, \xi_{T}\right] \mathscr{L}\left[\infty^{-}, \xi^{-}\right] \psi_{i}(\xi)\right| P\right\rangle,
$$

where $[+]$ denotes that the gauge link appearing in $\Phi$ is future-pointing, corresponding to the SIDIS process. The distribution $g^{\perp}$ can be deduced from $\Phi^{[+]}\left(x, k_{T}\right)$ by taking the trace with proper Dirac matrices

$$
-\frac{\varepsilon_{T}^{\alpha \rho} k_{T \rho}}{P^{+}} g^{\perp}\left(x, \boldsymbol{k}_{T}^{2}\right)=\frac{1}{2} \operatorname{Tr}\left[\Phi^{[+]} \gamma^{\alpha} \gamma_{5}\right] .
$$

Here, we extend the calculations in Ref. [8] using the spectator model with an axial-vector diquark to obtain $g^{\perp}$ for both the $u$ and $d$ quarks. We perform the calculation following the way used in [6] and choose the form for polarization sum of the axial-vector diquark $d_{\mu v}$ adopted in [7]. After performing the integrations over $k^{-}$and the momentum of the exchanged soft gluon to obtain the correlator in the spectator model, we arrive at the expressions [9] for the distribution $g^{\perp}$ from the scalar (denoted by $s$ ) and the axial-vector diquark components (denoted by $v$ ) by using Eq. (2.2),

$$
\begin{aligned}
g^{\perp s}\left(x, \boldsymbol{k}_{T}^{2}\right) & =-\frac{N_{s}^{2}(1-x)^{2}}{\left(32 \pi^{3}\right)} \frac{e_{s} e_{q}}{4 \pi}\left[\frac{(1-x) \Lambda_{s}^{2}+(1+x) M_{s}^{2}-(1-x) M^{2}}{L_{s}^{2}\left(L_{s}^{2}+\boldsymbol{k}_{T}^{2}\right)^{3}}\right], \\
g^{\perp v}\left(x, \boldsymbol{k}_{T}^{2}\right) & =\frac{N_{v}^{2}(1-x)^{2}}{\left(32 \pi^{3}\right)} \frac{e_{v} e_{q}}{4 \pi}\left[\frac{(1-x)(x M+m)^{2}+(1-x)^{2} M^{2}-M_{v}^{2}+x L_{v}^{2}}{(1-x) L_{v}^{2}\left(L_{v}^{2}+\boldsymbol{k}_{T}^{2}\right)^{3}}\right. \\
& \left.-\frac{x}{(1-x) \boldsymbol{k}_{T}^{2}\left(L_{v}^{2}+\boldsymbol{k}_{T}^{2}\right)^{2}} \ln \left(\frac{L_{v}^{2}+\boldsymbol{k}_{T}^{2}}{L_{v}^{2}}\right)\right] .
\end{aligned}
$$



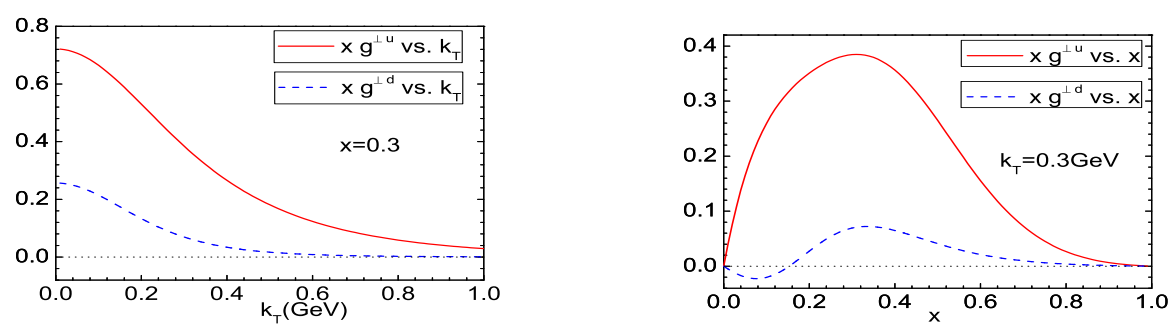

Figure 1: Left panel: model results for $x g^{\perp u}$ (solid line) and $x g^{\perp d}$ (dashed line) as functions of $k_{T}$ at $x=0.3$; right panel: model results for $x g^{\perp u}$ (solid line) and $x g^{\perp d}$ (dashed line) as functions of $x$ at $k_{T}=0.3 \mathrm{GeV}$.

The function $g^{\perp}$ for the $u$ and $d$ quarks can be constructed by $g^{\perp s}$ and $g^{\perp v}$ following the approach in Ref. [6]. For the parameters needed in the numerical calculation, we also adopt the fitted results from the same reference. The quark mass is chosen as $m=0.3 \mathrm{GeV}$. To convert our calculation to real QCD, we use the replacement $\frac{e_{q} e_{X}}{4 \pi} \rightarrow-C_{F} \alpha_{s}$ for the combination of the charges of the quark $q$ and the spectator diquark $X$, and choose $\alpha_{s} \approx 0.3$ in our calculation. In the left panel of Fig. 1, we plot the functions $x g^{\perp u}$ (solid line) and $x g^{\perp d}$ (dashed line) vs $k_{T}$ at $x=0.3$, while in the right panel, we display the $x$ dependence at $k_{T}=0.3 \mathrm{GeV}$. As we can see from Fig. 1 , the dominance of $u$ quark contribution is evident in the adopted spectator model, and the $x$ and $k_{T}$ dependencies of the $u$ and $d$ quark distributions are different.

\section{Numerical results for beam spin asymmetry}

The beam-spin asymmetry $A_{L U}^{\sin \phi}$ in single-pion production off an unpolarized target is expressed as

$$
A_{L U}^{\sin \phi}\left(P_{T}\right)=\frac{\int d x \int d y \int d z \frac{1}{x y Q^{2}} \frac{y^{2}}{2(1-\varepsilon)} \times\left(1+\frac{\gamma^{2}}{2 x}\right) \sqrt{2 \varepsilon(1-\varepsilon)} F_{L U}^{\sin \phi}}{\int d x \int d y \int d z \frac{1}{x y Q^{2}} \frac{y^{2}}{2(1-\varepsilon)} \times\left(1+\frac{\gamma^{2}}{2 x}\right) F_{U U}}
$$

for the $P_{T}$-dependent asymmetry. The $x$-dependent and the $z$-dependent asymmetries can be defined in a similar way. Here $F_{U U}$ and $F_{L U}^{\mathrm{sin} \phi_{h}}$ are the helicity-averaged and the helicity-dependent structure functions, respectively. In the parton model, the two structure functions in Eq. (3.1) can be expressed as the convolution of TMD DFs and FFs, based on the tree-level factorization adopted in Ref. [10].

To do the calculation on the beam SSA in semi-inclusive pion electroproduction contributed by the $g^{\perp} D_{1}$ term, we neglect the quark-gluon-quark correlators for FFs and consider merely the beam SSA of the $\pi^{0}$ production. Since the favored and the unfavored Collins functions have similar sizes but opposite signs [11], we have $H_{1}^{\perp \pi^{0} / q}=\left(H_{1}^{\perp \text { fav }}+H_{1}^{\perp \text { unf }}\right) / 2 \approx 0$ for the $\pi^{0}$ FF. Thus, in the following calculation, we can just take into account the term $g^{\perp} D_{1}$ and obtain

$$
F_{L U}^{\sin \phi_{h}} \approx \frac{2 M x}{Q} \sum_{q=u, d} e_{q}^{2} \int d^{2} k_{T}\left\{\frac{\hat{\boldsymbol{P}}_{T} \cdot k_{T}}{M}\left[x g^{\perp q}\left(x, k_{T}^{2}\right) \times D_{1}^{q}\left(z,\left(P_{T}-z k_{T}\right)^{2}\right)\right]\right\} .
$$

where $\hat{\boldsymbol{P}}_{T}=\frac{P_{T}}{P_{T}}$ with $P_{T}=\left|P_{T}\right|$. For the unpolarized TMDs $f_{1}^{q}\left(x, k_{T}^{2}\right)$, we adopt the results from the same spectator model calculation [6] for consistency. 

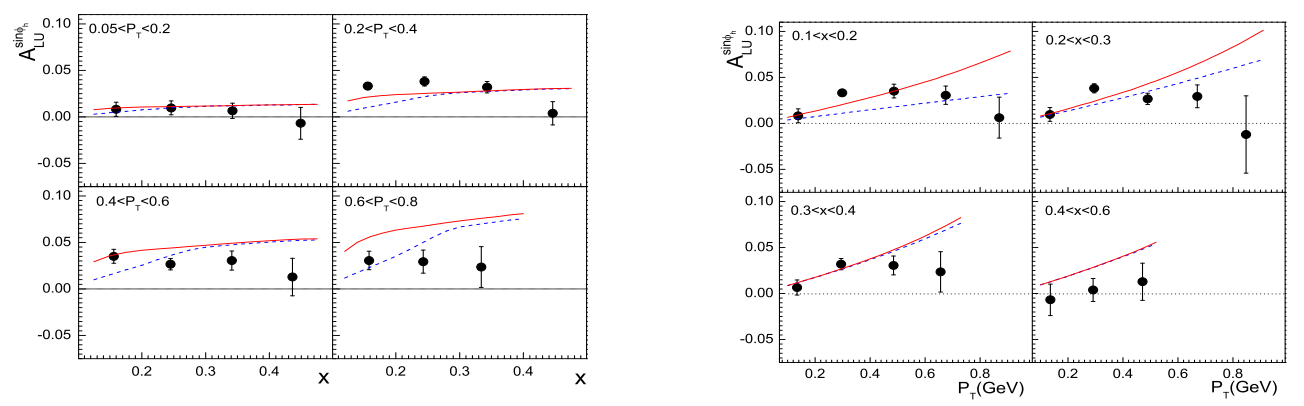

Figure 2: Beam SSA $A_{L U}^{\sin \phi_{h}}$ in $\pi^{0}$ electroproduction contributed by $g^{\perp}$. The solid and the dashed lines correspond to the results without and with the kinematical constraints on $k_{T}$, respectively.
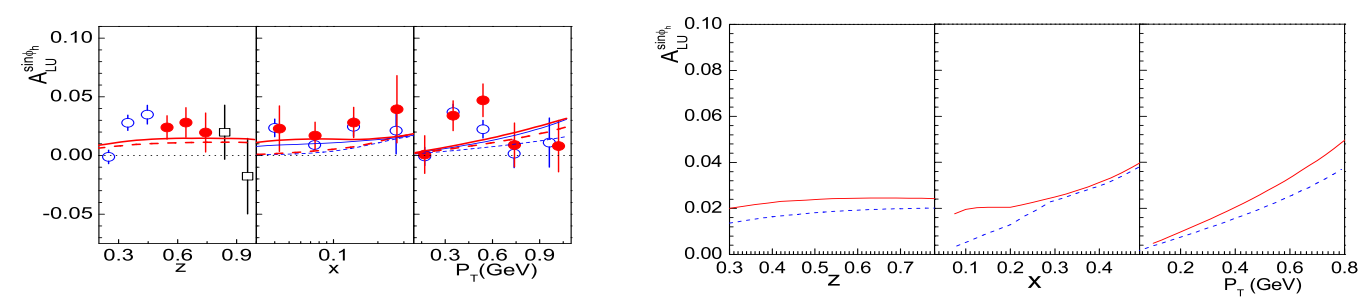

Figure 3: The beam SSA $A_{L U}^{\sin \phi_{h}}$ for $\pi^{0}$ production in SIDIS at HERMES (the left panel) and at CLAS12 (the right panel). The solid and the dashed lines correspond to the results without and with the kinematical constraints on $k_{T}$, respectively.

To perform the numerical calculation on the asymmetry $A_{L U}^{\sin \phi_{h}}$ in $\pi^{0}$ production at CLAS, we adopt the following kinematical cuts [3]:

$$
0.4<z<0.7, Q^{2}>1 \mathrm{GeV}^{2}, W^{2}>4 \mathrm{GeV}^{2}, P_{T}>0.05 \mathrm{GeV}, M_{x}\left(e \pi^{0}\right)>1.5 \mathrm{GeV},
$$

where $M_{x}\left(e \pi^{0}\right)$ are the missing-mass values for the $e \pi^{0}$ system. Finally, in our calculation, we consider the following kinematical constraints on the intrinsic transverse momentum of the initial quarks [12].

$$
\begin{cases}k_{T}^{2} \leq(2-x)(1-x) Q^{2}, & \text { for } 0<x<1 \\ k_{T}^{2} \leq \frac{x(1-x)}{(1-2 x)^{2}} Q^{2}, & \text { for } x<0.5\end{cases}
$$

The left panel of Fig. 2 shows the results [9] of the beam SSA $A_{L U}^{\sin \phi_{h}}$ for $\pi^{0}$ production as a function of $x$. In the right panel of Fig. 2, we display the same asymmetry as a function $P_{T}$. From the comparison between the theoretical calculation and the CLAS data, we conclude that the $g^{\perp} D_{1}$ term can account for the beam SSA in $\pi^{0}$ production at CLAS in the regions $x$ and $P_{T}$ are not large $\left(x<0.4\right.$ and $\left.P_{T}<0.5 \mathrm{GeV}\right)$.

Furthermore, we also compare our calculation of the $A_{L U}^{\sin \phi_{h}}$ with the data measured by the HERMES Collaboration using a $27.6 \mathrm{GeV}$ positron beam. In addition, the following kinematics are applied in the calculation [2]

$$
\begin{aligned}
& 0.023<x<0.4,0<y<0.85,1 \mathrm{GeV}^{2}<Q^{2}<15 \mathrm{GeV}^{2}, \\
& W^{2}>4 \mathrm{GeV}^{2}, \quad 2 \mathrm{GeV}<P_{h}<15 \mathrm{GeV},
\end{aligned}
$$


where $P_{h}$ is the energy of the detected final-state $\pi^{0}$ in the target rest frame. In the left panel of Fig. 3, we show the asymmetry and compare it with the HERMES data [2]. It is found that the theoretical curves agree with the experimental data within the statistical uncertainty, and the calculation without the constraints describes the data in the smaller $x$ region better.

Finally, we present the prediction for the asymmetry $A_{L U}^{\sin \phi_{h}}$ at CLAS12 (shown in the right panel of Fig. 3). The kinematical cuts applied in the calculation are

$$
\begin{aligned}
& 0.08<x<0.6,0.2<y<0.9,0.3<z<0.8, \\
& Q^{2}>1 \mathrm{GeV}^{2}, W^{2}>4 \mathrm{GeV}^{2}, 0.05 \mathrm{GeV}<P_{T}<0.8 \mathrm{GeV} .
\end{aligned}
$$

Our calculation shows that the beam SSA at CLAS12 is smaller than that at CLAS, but is still sizable. Also, our result indicates that there is no obvious $z$ dependence of the asymmetry in $\pi^{0}$ production. Therefore, the precision measurement of the $z$ dependence of $A_{L U}^{\sin \phi_{h}}$ in $\pi^{0}$ electroproduction at CLAS12 can verify the role of $g^{\perp}$ in beam SSA.

\section{Conclusion}

In this work, we have performed a calculation on the $T$-odd twist-3 TMD distribution $g^{\perp}\left(x, \boldsymbol{k}_{T}^{2}\right)$ for $u$ and $d$ quarks inside the proton in a spectator model with scalar and axial-vector diquarks. We found that $g^{\perp u}$ and $g^{\perp d}$ have different $x$ and $k_{T}$ dependencies. Using the model results, we analyzed the beam SSA $A_{L U}^{\sin \phi_{h}}$ in semi-inclusive pion electroproduction. The comparison between our theoretical calculation and the data indicates that the $g^{\perp} D_{1}$ term can account for the beam SSA in $\pi^{0}$ production measured by the CLAS Collaboration in the region $x<0.4$ and $P_{T}<0.5 \mathrm{GeV}$, where our theoretical curves describe the data fairly well. In addition, our calculated asymmetry at the HERMES kinematic region is consistent with the HERMES measurements after the error bars of the data are considered. Our study suggests that the $T$-odd twist- 3 distribution $g^{\perp}$ plays an important role in the beam SSA in SIDIS, especially in the case of neutral pion production.

\section{References}

[1] H. Avakian et al. (CLAS Collaboration), Phys. Rev. D 69112004 (2004).

[2] A. Airapetian et al. (HERMES Collaboration), Phys. Lett. B 648, 164 (2007).

[3] M. Aghasyan et al., Phys. Lett. B 704, 397 (2011).

[4] A. Metz and M. Schlegel, Eur. Phys. J. A 22, 489 (2004).

[5] A. Bacchetta, P.J. Mulders, and F. Pijlman, Phys. Lett. B 595, 309 (2004).

[6] A. Bacchetta, F. Conti, and M. Radici, Phys. Rev. D 78, 074010 (2008).

[7] S. J. Brodsky, D. S. Hwang, B. -Q. Ma, and I. Schmidt, Nucl. Phys. B593, 311 (2001).

[8] Z. Lu and I. Schmidt, Phys. Lett. B 712, 451 (2012).

[9] W. Mao and Z. Lu, Phys. Rev. D 87, 014012 (2013)

[10] A. Bacchetta, M. Diehl, K. Goeke, A. Metz, P. J. Mulders, and M. Schlegel, J. High Energy Phys. 02 093 (2007).

[11] A. V. Efremov, K. Goeke, and P. Schweitzer, Phys. Rev. D 73, 094025 (2006).

[12] M. Boglione, S. Melis, and A. Prokudin, Phys. Rev. D 84, 034033 (2011). 